\title{
Verwendung und Nachweis von Butterersatzstoffen in Backwaren.
}

\author{
Von \\ J. König und W. Burberg. \\ Mitteilung aus der Landwirtschaftlichen Versuchsstation Münter i. W. \\ [Eingegangen am 7. Januar 1914.]
}

In früheren Zeiten hat man für die Herstellung der Feinbackwaren, der sogenannten Teegebäcke, neben Wasser und Milch nur Butter, Mehl, Zucker und Eier verwendet. Nachdem aber die Nachfrage nach Fetten überhaupt ein Steigen der Preise für Butter als das geschätzteste Fett herbeigeführt und die Herstellung von Butterersatzstoffen in ausgedehntestem MaBe zur Folge gehabt hat, ist es von Belang, Umschau zu halten, wie es zurzeit mit der Verwendung von Fetten in der Feinbäckerei steht. Aus diesem Grunde haben wir von diesen Erzeugnissen die verschiedensten, billige und teure Sorten einer Untersuchung unterworfen.

$\mathrm{Zu}$ ihrer Herstellung kommen im großen und ganzen dieselben Stoffe zur Anwendung, wie bei der Bereitung von Honig- und Lebkuchen ${ }^{1}$ ). Jedoch tritt außer dem Zucker das Fett hier in den Vordergrund. Es werden zur Bereitung von Kakes, Waffeln usw. Butter und Mehl mit oder ohne Zusatz von Eiern zu einem Teige gerührt; der Teig wird dann, ohne gegoren zu haben, mit Zucker vermengt und in gewöhnlicher Weise gebacken. Heute werden diese Waren in sehr großem Maßstabe von Fabriken hergestellt, da sich der Kleinbetrieb kaum noch lohnt ${ }^{2}$ ).

Im Gegensatz hierzu ist die Herstellung der Blätterteigwaren auf den Kleinbetrieb beschränkt, da sie nur kurze Zeit haltbar sind. Zu ihrer Herstellung werden Butter und mit Wasser angerührtes Mehl lagenweise übereinandergeschichtet. Dann wird der Teig gezogen und ausgerollt, wozu eine besondere Fertigkeit gehört, und mit oder ohne aufgestreuten Zucker, Sirup, Fruchtgelee usw. in gewöhnlicher Weise gebacken. Eine besondere Art von mit Fett hergestelltem Gebäcke sind die unter dem Namen Spekulatius, Spekulatien oder Linzerteig hauptsäcblich in Nordwestdeutschland und dem nahen Holland zur Weihnachtszeit bereiteten Waren. Sie werden in ähnlicher Weise wie die Kakes hergestellt, sind aber im allgemeinen etwas mürber gebacken. Bei ihrer Herstellung herrscht im allgemeinen ebenfalls der Kleinbetrieb vor.

In den bereits oben ${ }^{1}$ ) angeführten Konditoreibüchern, denen auch vorstehende Angaben entnommen sind, ist stets von Butter die Rede, in der Praxis jedoch wird diese sehr häufig durch andere Fette ersetzt. Zu deren Nachweis ist der gegebene Weg, die analytischen Konstanten des aus dem Gebäck extrahierten Fettes festzustellen, doch ist die Frage von großer Bedentung, ob und inwieweit die Fettkonstanten durch das Fett der Zutaten, das Backen usw. beeinfluft werden. Die ersten Untersuchungen hierüber stellten E. Hofstädter ${ }^{4}$ ) und J. Gerum ${ }^{5}$ ) an,

1) Vergl. diese Zeitschrift 1914, 27, 761.

2) Eine der gröften Kakesfabriken, H. Bahlsen in Hannover, die unter andern die bekannten Leibniz-Kakes herstellt, beschäftigt 1600 Arbeiter und Angestellte; in einem Jahre werden dort angeblich etwa 2,7 Millionen Eier, 384000 Liter Milch, $180000 \mathrm{~kg}$ Butter, $2 \mathrm{Mil}$ lionen $\mathrm{kg} \mathrm{Mehl,} \mathrm{1,2} \mathrm{Millionen} \mathrm{kg}$ Zucker verarbeitet.

3) Diese Zeitschrift 1909, 17, 430.

4) Diese Zeitschrift 1912, 24, 513. 
deren Arbeiten bisher die einzigen größeren Mitteilungen in der Fachliteratur über die Buttergebäcke überhaupt sind.

Hofstädter bereitete aus Butter, Mehl und Zucker einen Teig, den er mit verschiedenen Zutaten beim Bäcker backen ließ. Bei der Untersuchung des mit Äther ausgezogenen Fettes prüfte er die Refraktometergrade, die Baudouin'sche und Soltsien'sche Reaktion und fand, daß auch bei kleinen Mengen Zutaten (Zimt, Eier, Vanillin, Muskatblüte, Safran, Citronenöl) die Soltsien'sche Reaktion positiv wurde, während die Baudouin'sche Reaktion stets negativ blieb; durch Eierzusatz trat eine erhebliche Erhöhung der Refraktometergrade ein. Bei Backversuchen mit Butter und Margarine in verschiedenen Mischungsverbältnissen zeigte sich deutlich, daß die Refraktometergrade nicht entscheidend für die Beurteilung sein können. Auch bier lieferte nur die Baudouin'sche Reaktion brauchbare Ergebnisse. Bei einem weiteren Backversuch stellte Hofstädter dann fest, daß auch die Reichert-MeiBl' sche Zahl und die Polenske'sche Zahl durch das Backen allein und durch die Zutaten verändert werden.

Aus $50 \mathrm{~g}$ Butter, $100 \mathrm{~g}$ Mehl und $25 \mathrm{~g}$ Zucker wurde ein Gebäck bereitet, dessen mit Äther ausgezogenes Fett folgende Konstanten zeigte :

Tabelle I.

\begin{tabular}{|c|c|c|c|c|c|}
\hline \multirow[b]{2}{*}{ Bezeichnung } & \multirow{2}{*}{$\begin{array}{l}\text { Refrakto- } \\
\text { metergrade } \\
40^{\circ} \mathrm{C}\end{array}$} & \multirow{2}{*}{$\begin{array}{l}\text { Reichert- } \\
\text { Meikl'sche } \\
\text { Zahl }\end{array}$} & \multirow[b]{2}{*}{$\begin{array}{l}\text { Polenske- } \\
\text { sche Zahl }\end{array}$} & \multicolumn{2}{|c|}{ Reaktionen auf Sesamöl } \\
\hline & & & & $\begin{array}{c}\text { nach } \\
\text { Baudouin }\end{array}$ & $\begin{array}{c}\text { nach } \\
\text { Soltsien }\end{array}$ \\
\hline Frtische Butter. & 41,8 & 29,3 & 2,5 & farblos & fablos \\
\hline Gebäck ohne Zutaten & 42,0 & 28,8 & 3,2 & , & rötlichgelb \\
\hline Gebäck mit $\mathrm{ki}$. . . & 44,2 & 25,9 & 2,6 & , & dunkelweinrot \\
\hline Gebäck mit $2 \mathrm{~g}$ Zimt & 42,2 & 28,8 & 2,3 & $"$ & hellweinrot \\
\hline
\end{tabular}

Auch bei der Untersuchung von 4 Proben aus Bäckereien, in denen laut Bèkanntmachung zur Herstellung aller Backwaren nur reine Naturbutter verwendet wurde, fand Hofstädter Refraktometergrade von 41,5 bis 44,2 , Reichert-MeiBI'sche Zahlen von 25,7 bis 29,3, Polenske'sche Zahlen von 2,6 bis 3,5, positive Soltsien'sche, aber stets negative Baudouin'sche Reaktionen.

Nach seinen Versuchen hält Hofstädter nur die Baudouin'sche Reaktion für zuverlässig bei Beurteilung von Buttergebäck und nimmt an, daß auch die Verseifungszahl und Jodzahl in ähnlich ungünstiger Weise beeinflußft werden. Ein ähnliches Ergebnis erhielt Schlegel ${ }^{1}$ ), als er aus Buttersehmalz, Mehl und Eiern ein Gebäck herstellte und das daraus abgeschiedene Fett untersuchte:

\begin{tabular}{|c|c|c|c|}
\hline & $\begin{array}{l}\text { Verseifungs" } \\
\text { zahl }\end{array}$ & $\begin{array}{c}\text { Reichert- } \\
\text { Meibl'scheZahI }\end{array}$ & $\begin{array}{l}\text { Refraktometer- } \\
\text { zahl bei } 40^{\circ}\end{array}$ \\
\hline Verwendetes Fett . . . . & 224,2 & 20,3 & 43,5 \\
\hline Aus dem Gebäck abgesehiedenes Fett & 219,2 & 18,7 & 46,0 \\
\hline
\end{tabular}

Diese beiden Mitteilungen veranlaßten J. Gerum, Versuche über den Einfluß von Eiern auf die Fettkonstanten anzustellen: Nach Literaturangaben wiegt ein Eigelb $16 \mathrm{~g}$ mit $33,0 \%$ Fett, ein Eigelb enthält also $5,33 \mathrm{~g}$ Fett; dieses hat die ReichertM e i 8 l' sche Zahl 0,66 und die Verseifungszahl 184,5-185,7. Verwendet man zu einem Backversuch auf $50 \mathrm{~g}$ Fett 1 oder 2 Eier, so kommen auf $50 \mathrm{~g}$ Fett rund $5-10 \mathrm{~g}$ Eifett, die die Reichert-Meißl'sche Zahl und die Verseifungszahl, wie folgt, beeinflussen:

1) Bericht über die Tätigkeit der Städtischen Untersuchungsanstalt für Nahrungs- und Genußmittel Nürnberg, 1911, S. 21. 
Tabelle II.

\begin{tabular}{c|c|c|c|c|c|c}
\hline \multirow{2}{*}{$\begin{array}{c}\text { Art des } \\
\text { Fettes }\end{array}$} & \multicolumn{2}{|c|}{ Reichert-Meikl'sche Zahl } & \multicolumn{3}{c}{ Verseifungszahl } \\
\cline { 2 - 4 } & Fett allein & $+1 \mathrm{Ei}$ & +2 Eier & Fett allein & $+1 \mathrm{Hi}$ & +2 Eier \\
\hline Butter . . & 28,93 & 26,38 & 24,23 & 228,8 & 225,9 & 221,5 \\
Palmin . . & 7,75 & 7,11 & 6,40 & 255,5 & 249,3 & 243,7 \\
Margarine . & 1,87 & 1,76 & 1,67 & 196,9 & 196,0 & 193,9
\end{tabular}

In der Praxis liegen die Verhältnisse für den Nachweis von Butterersatzfett insofern günstiger, als auf gleiche Mengen Fett weniger Eier kommen, zumal auch nach $\mathrm{H}$ of städter die Baudou in'sche Reaktion nicht beeinflußt wird.

Eine weitere Fehlerquelle sieht Gerum in der Extraktion mit Äther, weil dadurch unverseifbare Bestandteile mitgelöst werden. Spaeth ${ }^{1}$ ) hat schon früher die Reinigung des Ätherextraktes mit niedrig siedendem Petroläther empfohlen. Gerum gibt' ein Verfahren an, das nicht quantitativ sei, aber gelbe, klar verseifbare Fette liefere: In einem großen Kolben wird $\mathbf{1} / \mathbf{2}$ bis 1 Liter Wasser zum Sieden erhitzt, darin das zerkleinerte Backwerk eingetragen und auf dem Wasserbad belassen, bis ein dicklicher Brei entstanden ist. Dann wird der Kolben mit kochendem Wasser bis oben an den Rand gefüllt, worauf sich das Fett an der Oberfläche abscheidet. Das Fett wird abgenommen, in Petroläther gelöst, filtriert, getrocknet und kann für weitere Bestimmungen verwendet werden. Gerum ließ aus $50 \mathrm{~g}$ Fett, $25 \mathrm{~g}$ Zucker und $200 \mathrm{~g}$ Mehl mit und ohne Zusatz von Eiern aus Butter, Palmin und Margarine Gebäcke herstellen und untersuchte diese nach dem alten sowie nach seinem neuen Verfahren. Er fand aber stets andere Reichert-MeiBl'sche Zahlen und Verseifungszahlen, als die ursprünglichen Fette aufwiesen, aber auch fast stets niedrigere Zahlen, als er aus dem Eizusatz berechnet hatte; die Schwankungen sind aus folgender Tabelle ersichtlich:

Tabelle IIr.

\begin{tabular}{|c|c|c|c|c|c|c|c|c|}
\hline \multirow{3}{*}{$\begin{array}{c}\text { Art } \\
\text { des Fettes }\end{array}$} & \multicolumn{2}{|c|}{ Konstanten des Fettes } & \multicolumn{2}{|c|}{ Gebäck ohne Ei } & \multicolumn{4}{|c|}{ Gebäck mit 2 Eiern } \\
\hline & \multirow{2}{*}{$\begin{array}{l}\text { Reichert- } \\
\text { Meisl'sche } \\
\text { Zahl }\end{array}$} & \multirow{2}{*}{$\begin{array}{c}\text { Verseifungs- } \\
\text { zahl }\end{array}$} & \multirow{2}{*}{$\begin{array}{l}\text { Reichert- } \\
\text { Meiflsehe } \\
\text { Zahl }\end{array}$} & \multirow{2}{*}{$\begin{array}{c}\text { Verseifungs- } \\
\text { zahl }\end{array}$} & \multicolumn{2}{|c|}{$\begin{array}{c}\text { Reichert- } \\
\text { MeiBl'seheZahl }\end{array}$} & \multicolumn{2}{|c|}{ Verseifungszahl } \\
\hline & & & & & ber. & gef. & ber. & gef. \\
\hline Butter & $2 Q, 9$ & 228,8 & 28,6 & 228,8 & 24,2 & $26,2-22,8$ & 221,5 & $218,4-214,8$ \\
\hline Palmin . & 7,8 & 255,5 & 7,7 & 255,5 & 6,4 & $7,7-7,0$ & 243,7 & $251,7-235,1$ \\
\hline Margarine & 1,9 & 196,9 & 1,8 & 196,9 & 1,7 & $1,7-1,5$ & 193,9 & $192,7-186,8$ \\
\hline
\end{tabular}

Bei einem Gebäck aus Butter mit der Reichert-Meißl'schen Zahl 20,2 und der Verseifungszahl 220,5 und einem Ei fand er die Reichert-Meißl'sche Zahl 17,2 statt der berechneten 18,5 und die Verseifungszahl $214,5-210,5$ statt der berechneten 217,4, also Zahlen, die sonst für Butter zweifelhaft erscheinen. Auch er empfiehlt des. halb Vorsicht bei der Beurteilung von Buttergebäcken auf Grund der Fettkonstanten.

Für unsere eigenen Versuche verfuhren wir zur Gewinnung des Fettes noch anders, weil das Gerum'sche Verfabren nach unseren Erfahrungen zu große Mengen Ausgangsmaterial erfordert, da wenigstens $20 \mathrm{~g}$ Fett zur vollständigen Untersuchung notwendig sind: In einen 1 Liter fassenden Kolben wurde in etwa 1/2 Liter Wasser soviel des Gebäckes eingetragen, dab die Ausbeute an Fett etwa $25 \mathrm{~g}$ betrug, und

1) Zeitschr, angew. Chemie 1894, 7, 294.

N. 14. 
diese Menge wurde mit 20 ccrn Salzsäure (spez. Gew. 1,124) zur Löslichmachung der Stärke 2 Stunden im Wasserbade mit aufgesetztem Küblrohr erhitzt, Nach dem Erkalten und Neutralisieren wurde der Brei in einem geräumigen Schütteltrichter mehrere Male mit Petroläther ausgeschüttelt. Von den vereinigten Lösungen wurde der Petroläther abdestilliert, das Fett in Kohlensäurestrom getrocknet und in mit

Tabelle

Buttergebäcke und

\begin{tabular}{|c|c|c|c|c|c|c|c|c|c|c|c|c|c|c|c|c|c|}
\hline \multirow[b]{2}{*}{ : } & \multirow{2}{*}{\multicolumn{2}{|c|}{ Nähere Bezeichnung }} & \multirow[b]{2}{*}{ 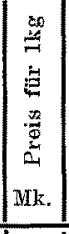 } & \multicolumn{12}{|c|}{ In der natürlichen Substanz } & \multirow{2}{*}{\multicolumn{2}{|c|}{\begin{tabular}{|c|} 
Polarisation \\
der \\
$10 \%$-igen \\
Lösung
\end{tabular}}} \\
\hline & & & & 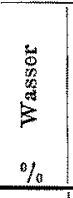 & 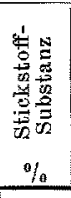 & $\%$ & 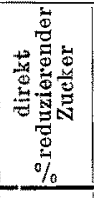 & 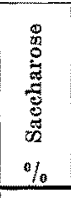 & 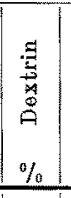 & 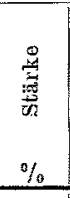 & 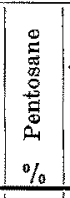 & 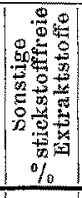 & 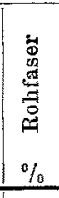 & $\begin{array}{l}\frac{2}{0} \\
\% \\
4 \\
\end{array}$ & 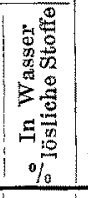 & & \\
\hline 1 & (Nr. 1 & (Butter) & & 4,47 & 9,58 & 24,95 & 1,02 & 9,94 & 2,58 & 42,62 & 2,41 & 1,44 & 0,40 & 0,59 & 12,69 & $+1,50$ & $-0,12$ \\
\hline 2 &, 2 & (Margarine) & . & 5,04 & 9,83 & 25,04 & 0,39 & 10,80 & 3,12 & 40,56 & 2,47 & 1,71 & 0,40 & 0,64 & 12,13 & $+1,55$ & $-0,15$ \\
\hline 5 & $" 31$ & (Cocosfett). & . & 3,23 & 9,22 & 27,59 & 0,46 & 9,95 & 2,58 & 39,69 & 1,94 & 4,59 & 0,17 & 0,48 & 11,20 & $+1,60$ & $-0,17$ \\
\hline 4 & $\stackrel{9}{=}=4$ & $\begin{array}{l}\text { Butter u. Mar- } \\
\text { garine . . }\end{array}$ & & 1,94 & 7,56 & 29,53 & 2,41 & 9,93 & 2,00 & 40,36 & 8,06 & 2,27 & 0,44 & 0,50 & 13,44 & $+1,65$ & $-0,20$ \\
\hline 5 & 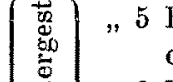 & $\begin{array}{l}\text { Butter und Co- } \\
\text { cosfett . . }\end{array}$ & . & 1,94 & 7,83 & 29,63 & 0,83 & 9,79 & 1,18 & 42,31 & 2,88 & 2,74 & 0,54 & 0,38 & 11,69 & $+1,58$ & $-0,20$ \\
\hline 6 & 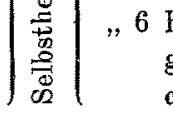 & $\begin{array}{l}\text { Butter u. Mar- } \\
\text { garine und } 0^{-} \\
\text {cosfett. . . }\end{array}$ & & 2,41 & 7,89 & 26,88 & 1,25 & 10,02 & 1,58 & 41,85 & $|3,58|$ & 2,45 & 1,65 & 0,49 & 12,50 & $+1,58$ & $-0,22$ \\
\hline 7 & Leibniz -Waff & ffeln . . & $4,40 \mid$ & 6,95 & 8,53 & 17,99 & $\left.5,6^{1}\right)$ & 80,76 & 8,50 & 12,10 & 2,11 & 4,75 & 1,47 & 1,15 & 39,10 & $+3,92$ & $-1,28$ \\
\hline 8 & Runde Füllh & hippen . . & 4,40 & 5,74 & 7,20 & 24,51 & $\left.14,10^{2}\right)$ & 27,91 & 6,70 & 8,64 & 2,13 & 0,79 & 1,08 & 1,20 & 44,70 & $+2,64$ & $-2,44$ \\
\hline 9 & Flache Fürst & ten waffeln & 4,40 & 2,50 & 5,75 & 33,83 & 1,41 & 29,20 & 7,65 & 13,04 & 1,75 & 0,91 & 2,71 & 1,22 & 42,50 & $+3,16$ & $-2,00$ \\
\hline 10 & Champagner & Vaffeln & $4,00 \mid$ & 7,01 & 9,26 & 4,63 & 2,50 & 42,83 & 4,20 & 25,28 & 2,06 & 1,36 & 0,31 & 0,5 & 46,00 & $+6,48$ & $-1,12$ \\
\hline 11 & Pangani- $\mathrm{n} . \mathrm{V}$ & Wiener Mischg. & 3,60 & 10,17 & 5,26 & 17,84 & 1,47 & 30,75 & 2,32 & 27,29 & 1,63 & \begin{tabular}{|l|l|}
3 & 1,57
\end{tabular} & 0,83 & 0,8 & $|39,80|$ & $+4,16$ & $-0,96$ \\
\hline 12 & Marienburge & er Mischung . & $3,00 \mid$ & 6,75 & 10,58 & 13,49 & 1,20 & 30,67 & 3,68 & 25,96 & 2,01 & 4,02 & 0,82 & 0,8 & 37,40 & $+3,92$ & $\mid-1,16$ \\
\hline 13 & Steinhuder & Mischang . & 2,60 & 9,80 & 7,56 & 8,68 & 2,70 & 27,68 & 2,56 & 34,84 & 42,66 & 6) 1,98 & 0,78 & 30,76 & 35,70 & $+3,88$ & $-1,00$ \\
\hline 14 & Leibniz-Kake & $\operatorname{ces} .$. & 2,40 & 7,78 & 7,68 & 12,03 & 2,21 & 17,01 & 8,08 & 43,11 & 2,85 & $5 \mid 2,12$ & 0,98 & 1,15 & 24,00 & $-2,08$ & -0.76 \\
\hline 15 & Kolonial-Kak & kes. . & 2,00 & 4,01 & 6,97 & 17,40 & 2,56 & 18,89 & 3,18 & 40,50 & 3,38 & \begin{tabular}{l|l}
8 & 1,90
\end{tabular} & 0,52 & 0,69 & 24,35 & $+2,58$ & $-0,88$ \\
\hline 16 & Demilunen - $V$ & Vanille & 2,00 & 8,37 & 7,32 & 8,02 & 3,76 & 19,62 & 3,53 & & 32,58 & 0,98 & 0,32 & 0 & 25,90 & $+2,78$ & $-0,70$ \\
\hline 17 & Lebkuchen-K & Konfekt . & 1,60 & 3,94 & 7,44 & 11,09 & $9,56^{3}$ & 22,54 & 3,9 & 33,46 & 62,71 & 13,57 & 0,85 & 0 & 34,10 & $+4,20$ & $+0,42$ \\
\hline 18 & Kaiser-Misch & hung . . & 1,40 & 5,80 & 7,32 & 12,04 & 1,50 & $25,7 \varepsilon$ & 33,90 & 38,58 & 32,71 & 0,79 & 0,95 & 0,68 & 29,25 & $+3,80$ & $-0,63$ \\
\hline 19 & Figaro-Misch & hang & 1,20 & 3,94 & 7,44 & 6,14 & 1,68 & 15,38 & 4,05 & 54,44 & 43,13 & 2,58 & 0,81 & 0,91 & 20,10 & $+1,75$ & $-0,92$ \\
\hline 20 & & $\mathrm{M}$ & 2,20 & 7,81 & 5,19 & 37,41 & 2,30 & 11,31 & 2,99 & 27,76 & 1,88 & \begin{tabular}{l|l}
8 & 1,98
\end{tabular} & 0,20 & 1,17 & 15,45 & $+1,56$ & $-0,31$ \\
\hline 21 & Blätter- & $E$ & 1,00 & 5,26 & 4,55 & 29,46 & 3,90 & $25,2:$ & 32,78 & 20,08 & 84,33 & \begin{tabular}{l|l}
8 & 0,58
\end{tabular} & 2,28 & 1,54 & $\mid 32,86$ & $+3,61$ & $-0,24$ \\
\hline & teig. & Sch. & 2,00 & 5,68 & 5,45 & 33,82 & 4,96 & 12,04 & 3,74 & 27 & 2,14 & 4) 1,30 & 1,34 & 1,70 & 20,37 & $+2,64$ & $=-$ \\
\hline 23 & waren & B. & 2,80 & $7,71^{\prime}$ & 6,60 & 29,67 & 2,00 & 17,97 & 3,91 & 28,24 & 42,00 & \begin{tabular}{l|l}
0,19 \\
0,19
\end{tabular} & 0,57 & 1,10 & 24,41 & $+2,46$ & $-0,75$ \\
\hline 24 & & H & 3,20 & 5,03 & 9,65 & 45,06 & - & 2,55 & - & 33,28 & $8-$ & 3,07 & 0,30 & 1,06 & -1 & - & - \\
\hline 25 & & & 2,00 & 4,80 & 9,81 & 16,67 & $\begin{array}{l}1,78 \\
\end{array}$ & 25,25 & 2,08 & 33,46 & 3,86 & 0,31 & 0,98 & 1,05 & 31,55 & $+3,45$ & $-0,93$ \\
\hline 2 & & & 1,60 & 5,46 & 8,38 & 8,80 & 1,66 & 22,68 & 1,38 & 40,49 & 3,96 & \begin{tabular}{l|l}
5,92 \\
5,92
\end{tabular} & 0,50 & 0,69 & 36,25 & $+3,92$ & $\pm 0,00$ \\
\hline 2 & & & 1,60 & 4,06 & 9,00 & 11,34 & 1,03 & 27,68 & 1,46 & 40,39 & 3,55 & \begin{tabular}{|l|l|}
5 & 0,44
\end{tabular} & 0,20 & 0,85 & 33,60 & $+3,70$ & $-1,08$ \\
\hline & & & 1,20 & 5,09 & 8,94 & \begin{tabular}{|l|}
9,39 \\
\end{tabular} & 0,80 & 27,23 & 1,75 & 41,55 & 3,70 & 0,51 & 0,22 & 0,73 & 34,62 & $+3,60$ & $-1,05$ \\
\hline & spekulatıus & & 1,60 & 5,87 & 7,94 & 12,07 & 1,01 & 27,43 & 1,15 & 40,58 & 2,60 & 0,20 & 0,33 & 0,82 & 33,54 & $+3,50$ & $-0,98$ \\
\hline & & & 1,20 & 5,90 & 8,00 & 9,88 & 0,90 & 25,85 & 2,00 & 40,78 & 3,92 & 1,65 & 0,27 & 0,85 & 37,52 & $+3,50$ & $-1,05$ \\
\hline & & & 1,60 & 4,10 & 7,69 & 14,81 & 2,85 & 24,90 & 1,85 & 37,60 & 3,59 & 0,69 & 0,73 & 1,19 & 32,85 & $+3,30$ & $-0,90$ \\
\hline & & & 1,20 & 4,89 & 9,00 & 11,98 & 1,62 & 19,19 & 1,85 & 44,73 & 3,60 & 1,32 & 0,75 & 1,07 & 26,65 & $+2,55$ & $-0,55$ \\
\hline
\end{tabular}


Gummipfropfen verschlossenen Kölbchen aufbewahrt. Bei jedem Fett wurden bestimmt: 1. die Reichert-Meißl'sche und die Polenske'sche Zahl, 2. die Verseifungszahl, 3. die Jodzahl nach Wijs, 4. die Refraktionsżahl bei $40^{\circ}$ und 5 . die $\mathrm{B}$ a u do u in'sche Reaktion ausgeführt.

Die Ergebnisse der Untersuchung sind in der Tabelle IV enthalten.

IV.

Butterteigwaren.

\begin{tabular}{|c|c|c|c|c|c|c|c|c|c|c|c|c|c|c|c|c|c|c|c|c|}
\hline \multirow[b]{2}{*}{ 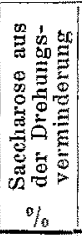 } & \multirow[b]{2}{*}{ 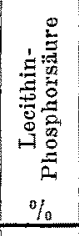 } & \multicolumn{11}{|c|}{ In der Trockensubstanz } & \multirow[b]{2}{*}{ 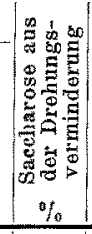 } & \multirow[b]{2}{*}{ 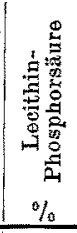 } & \multicolumn{5}{|c|}{ Konstanten des Fettes } & \multirow[b]{2}{*}{ 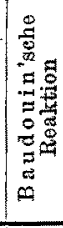 } \\
\hline & & 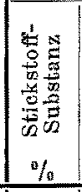 & $\begin{array}{l}\overrightarrow{0} \\
\% \\
0\end{array}$ & 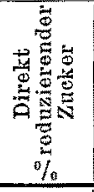 & 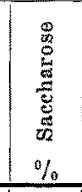 & 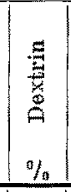 & 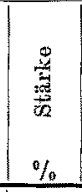 & 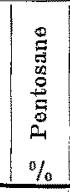 & 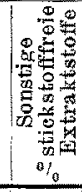 & 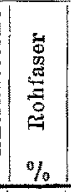 & $\begin{array}{l}\frac{0}{3} \\
\frac{8}{4} \\
\% \\
\% \\
\end{array}$ & 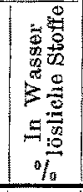 & & & 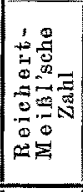 & 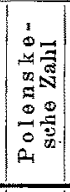 & 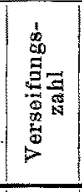 & 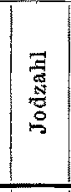 & 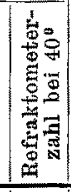 & \\
\hline 9,2 & 0,080 & $|10,03|$ & 26,12 & 1,07 & 10,40 & 2,70 & 44,61 & 2,52 & 1,51 & 0,42 & 0,62 & 13,28 & 9,69 & 0,080 & 27,4 & 3,2 & 220,9 & 385,6 & 35,3 & 0 \\
\hline $9, \dot{4}$ & 0,075 & $|10,36|$ & 26,37 & 0,41 & 11,37 & 3,29 & 42,71 & $\mid 2,60$ & 1,80 & 0,42 & 0,67 & 12,77 & 9,96 & 0,075 & 3,1 & 2,8 & 187,5 & 560,2 & 49,3 & + \\
\hline 10,09 & 0,068 & 9,53 & 28,51 & 0,48 & 10,28 & 2,67 & 41,01 & 2,01 & 4,83 & 0,18 & 0,50 & 11,57 & 10,43 & 0,068 & 6,7 & 16,2 & 264,7 & 713,0 & 38,0 & 0 \\
\hline 10,54 & 0,018 & 7,71 & 30,11 & 2,46 & 10,13 & 2,04 & 41,16 & 3,12 & 2,31 & 0,45 & 0,51 & 13,71 & 10,72 & 0,018 & 12,1 & 2,8 & 196,7 & 52,1 & 47,0 & + \\
\hline 10, & $\begin{array}{l}0,026 \\
0,029\end{array}$ & 7,98 & 30,22 & 0,85 & 9,98 & 1,20 & 49,15 & $5 \mid 2,89$ & 2,79 & 0,55 & 0,39 & 11,92 & 10,32 & $\begin{array}{l}0,026 \\
0,029\end{array}$ & $-12,9$ & 10,6 & 252,3 & 19,3 & 340,0 & 0 \\
\hline 10,26 & 0,025 & 8,09 & 27,54 & 1,28 & 10,27 & $|1,62|$ & 42,88 & 33,62 & 2,51 & 1,69 & 0,50 & 12,81 & 10,55 & 0,025 & 12,7 & 6,6 & 232,8 & 336,3 & 43,0 & + \\
\hline 29,64 & 0,042 & 9,17 & 19,33 & $\left.6,12^{1}\right)$ & 33,06 & 9,14 & 13,01 & 2,27 & 7) 5,08 & 1,58 & 1,24 & 42,02 & 31,81 & 0,042 & 3,7 & 1,9 & 190,8 & 307,0 & 51,5 & 0 \\
\hline 2 & 0,053 & 7,63 & 25,97 & $\left(14,94^{2}\right)$ & 29,57 & 7,10 & 9,16 & $3 \mid 2,26$ & \begin{tabular}{|l|l}
6 & 0,96
\end{tabular} & 1,14 & 1,27 & 47,37 & $80, \pi$ & 0,053 & 2,1 & 0,8 & 204,8 & 50,3 & 50,2 & 0 \\
\hline 2 & 0,014 & 5,90 & 34,70 & 1,44 & 29,98 & 7,85 & 13,37 & 7,80 & 0,93 & 2,78 & 1,25 & 48,6 & 230,1 & 0,014 & 1,3 & 3,0 & 189 & $\mid 32,6$ & 47,0 & \\
\hline 4 & 0,161 & 9,96 & 4,98 & 2,69 & 46,06 & 4,52 & 27,18 & 32,22 & 1,46 & 0,33 & 0,60 & 49,47 & 746,57 & 0,161 & 0,6 & 0,8 & 182,2 & 63,5 & 55,1 & 0 \\
\hline 18 & 0,043 & 5,86 & 19,86 & 1,64 & 34,22 & 2,58 & 30,38 & 81,81 & 1,76 & 0,92 & 0,97 & 44,30 & 82,49 & 0,043 & 27,1 & 2,0 & 232,1 & 40,2 & 46,2 & 0 \\
\hline & 0,073 & 11,35 & 14,47 & 1,29 & 32,89 & 3,95 & 27,81 & 2,16 & \begin{tabular}{|l|l}
5 & 4,29
\end{tabular} & 0,88 & 0,91 & 40,11 & 131,12 & 0,078 & 9,4 & 1,3 & 207,2 & 65,3 & 50,5 & 0 \\
\hline & 0,043 & 8,38 & 9,62 & 2,99 & 30,68 & 2,88 & 88,63 & 2,95 & 2,20 & 0,87 & 0,85 & 39,58 & 830,84 & 0,043 & 7,9 & 2,5 & 211,5 & 52,9 & 46,0 & 0 \\
\hline & 0,033 & 8,33 & 13,04 & 2,40 & 18,44 & $\{3,26$ & 46,75 & 53,09 & 2,30 & 1,06 & 1,2 & 26,02 & 17,5 & 0,033 & 22,6 & 3,4 & 217 & 42,7 & 47,2 & \\
\hline & 0,043 & 7,26 & 18,13 & 2,67 & 19,68 & 3,31 & 42,19 & 33,52 & \begin{tabular}{l|l} 
2) 1,98
\end{tabular} & 0,54 & 0,7 & & 72 & 0,043 & 4,8 & 6,7 & 250,9 & 13,4 & 88,5 & 0 \\
\hline & 0,025 & 7,99 & 8,75 & 4,10 & 21,41 & 3,85 & 48,93 & 32,76 & 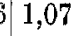 & 0,35 & 0 & & & 0,025 & 3,7 & 6,6 & 247,4 & $\mid 22,5$ & 38,6 & 0 \\
\hline & 0,026 & 7,75 & 11,54 & $\left.9,95^{3}\right)$ & 23,47 & 4,14 & 34,83 & 2,83 & 3,71 & 0,89 & 0,90 & 5,50 & 22,40 & 0,026 & 0,6 & 1,2 & 197,7 & 47,3 & 47,0 & 0 \\
\hline & 0.039 & 7,70 & 12,78 & 1,59 & 27,37 & 4,14 & 40,90 & 2,88 & \begin{tabular}{|l|l|}
3 & 0,84
\end{tabular} & 1,01 & 0,72 & 31,05 & 26,79 & 0,039 & 2,3 & 2,0 & 213,0 & 61,7 & $7.53,2$ & 0 \\
\hline & 0,050 & 7,75 & 0,39 & 1,75 & 16,01 & 4,22 & 56,54 & 43,26 & 2,81 & 0,32 & 0,95 & $\{20,92$ & & 0,050 & 3,5 & 5,4 & 259,6 & 19,2 & 40,7 & 0 \\
\hline, 66 & - & 5,63 & 40,58 & 2,50 & 12,27 & 3,24 & 30,11 & 2,04 & $\begin{array}{l}4,16 \\
\end{array}$ & 0,21 & 1,26 & 16,76 & \begin{tabular}{l|l}
6 & 11,54
\end{tabular} & - & 0,0 & 1,7 & 196 , & 64,1 & 53,5 & + \\
\hline 95 & - & 4,81 & 31,10 & 4,12 & 26,63 & 2,94 & 21,20 & 4,57 & 0,61 & 2,41 & 1,63 & 34,68 & 23,14 & - & 0,2 & 5,5 & 201,1 & 63,9 & 51,7 & + \\
\hline- & - & 5,78 & 35,83 & 5,26 & 12,76 & 3,96 & 29,50 & 2,27 & 1,39 & 1,42 & 1,80 & 21,59 & 9 & 一 & 20,4 & 1,4 & 216,8 & 324,2 & 24,2 & 0 \\
\hline 18,80 & - & 7,16 & 32,16 & 2,17 & 19,48 & 4,23 & 30,61 & 2,17 & 0,21 & 0,62 & 1,19 & 26,46 & $6[19,83$ & - & 20,9 & 3,0 & 213,6 & 27,6 & 47,0 & 0 \\
\hline- & 0,046 & 10,16 & 47,45 & - & 2,68 & - & 35,04 & $1-$ & 3,23 & 0,32 & 1,12 & - & - & 0,046 & 12,3 & $1, \mathrm{~s}$ & 209,7 & 51,5 & 48,1 & + \\
\hline 24,97 & - & 10,31 & 17,51 & 1,87 & 26,52 & 2,19 & 35,14 & 4,16 & 0,33 & 0,98 & 1,10 & 38,14 & 426,22 & - & 17,0 & 3,1 & 214,0 & 55,8 & 39,5 & 0 \\
\hline & - & 8,87 & $9, \mathbf{3 1}$ & 1,77 & 23,99 & 1,46 & 42,83 & 4,19 & 6,32 & 0,58 & $0,70^{\circ}$ & 38,34 & 423,66 & - & 24,6 & 5,5 & 227,1 & 39,3 & 45,5 & 0 \\
\hline 25 & - & 9,34 & 11,82 & 1,07 & 28,86 & 1,52 & 42,10 & 3,70 & 0,49 & 0,21 & 0,8 & 35,02 & 28,39 & - & 13,6 & 2,7 & 21 & 48,0 & 48,0 & + \\
\hline ;,51 & - & 9,42 & 9,90 & 0,84 & 28,70 & 1,84 & 43,77 & 3,90 & 0,63 & 0,23 & 0,77 & 36,48 & 327,87 & - & 3,1 & 1,4 & 195,1 & 67,0 & 51,5 & + \\
\hline 6,54 & - & 8,43 & 12,82 & 1,08 & 29,15 & 1,25 & 43,08 & 32,76 & 0,21 & 0,35 & 0,87 & 85,68 & 27,13 & - & 2,9 & 1,5 & 209,8 & 367,0 & 58,5 & + \\
\hline, 94 & - & 8,50 & 10,50 & 0,96 & 27,47 & 2,14 & 43,12 & 4,17 & 1,71 & 0,29 & 1,14 & 39,87 & 727,59 & - & 4,5 & 1,8 & 200,4 & 472,3 & 54,8 & + \\
\hline 3,94 & - & 8,02 & 15,80 & 2,97 & 25,97 & 1,93 & 38,82 & 3,74 & 0,75 & 0,76 & 1,24 & 34,26 & 24,97 & - & 4,5 & 1,5 & 199,4 & 70,5 & 54,5 & + \\
\hline 17,67 & - & 9,46 & 12,60 & 1,70 & 20,17 & 1,95 & 47,03 & 3,79 & 1,38 & 0,79 & 1,13 & 28,02 & 18,58 & - & 1,9 & 0,8 & 208,9 & 71,5 & 54,8 & 0 \\
\hline
\end{tabular}


Um einen Zusatz von Eiern festzustellen, wurde nach dem von Juckenack ${ }^{1}$ ) für Eierteigwaren angegebenen Verfahren die Lecithin-Phosphorsäure bestimmt, wobei wir uns bewußt waren, daß infolge des Lecithin-Gehaltes der Fette, besonders der Butter, nicht genau die von Juckenack für eine bestimmte Menge Eier auf ein Pfund Mehl angegebenen Zahlen erhalten werden konnten.

Die anderen Bestandteile wurden teils nach den früher ${ }^{2}$ ) beschriebenen, teils nach den üblichen Verfabren ermittelt. Betreffs der quantitativen Fettbestimmung ist zu bemerken, daß bei diesen Erzeugnissen das Gefüge so locker ist, daß eine Extraktion im Soxhlet-Apparat nach Verreiben der Substanz mit Sand genügt und sich die etwas umständliche Arbeit im v. d. Heide $e^{7}$ schen Apparat erübrigt. Kontrollversuche nach beiden Verfahren zeigten übereinstimmende Ergebnisse.

Da hauptsächlich für die Herstellung der Feinbackwaren Butter, Cocosfett (Palmin) und Margarine verwendet werden, wurden zunächst die unter No. 1 bis 6 (S. 778- 779.) aufgeführten mit den genannten Fetten und Mischungen aus ihnen selbst hergestellten Gebäcke untersucht. Die verwendeten Fette zeigten folgende Konstanten:

Tabelle V.

\begin{tabular}{l|c|c|c|c|c|c}
\hline \hline $\begin{array}{c}\text { Art } \\
\text { des Fettes }\end{array}$ & $\begin{array}{c}\text { Reichert- } \begin{array}{c}\text { Meißl'sche } \\
\text { Zahl }\end{array} \\
\text { Polenske' } \\
\text { sche Zahl }\end{array}$ & $\begin{array}{c}\text { Verseifungs- } \\
\text { zahl }\end{array}$ & Jodzahl & $\begin{array}{c}\text { Refrakto- } \\
\text { meterzahl } \\
40^{\circ}\end{array}$ & $\begin{array}{c}\text { Baudouin'- } \\
\text { sche Reaktion }\end{array}$ \\
\hline Butter . . & 33,1 & 2,4 & 225,4 & 30,3 & 44,8 & negativ \\
Margarine & 2,3 & 2,5 & 203,8 & 56,4 & 49,2 & positiv \\
Cocosfett . & 8,2 & $\mathbf{1 6 , 4}$ & 251,2 & 6,4 & 37,9 & negativ
\end{tabular}

Ein Vergleich der Konstanten der gewonnenen Fette mit denen der Ausgangsfette lehrt, dafo tatsächlich die Zahlen andere sind, doch sind die Abweichungen nicht derart, daß man nicht aus dem Gesamtbild der Analyse das verwendete Fett bezw. die Fettmischung erkennen könnte.

Bei den anderen Proben No. 7-32 zeigt sich dann, daß von diesen $26 \mathrm{Ge}-$ bäcken nur 5 allein mit Butter gebacken sind, nämlich No. 11, 14, 22, 23 und 26, letztere vielleicht mit Cocosfettzusatz. No. 20,21, 27, 28, 29, 30, 31 sind zweifellos mit Margarine gebacken, No. 24 mit einem Gemisch von Butter und Margarine, No. 25 mit einem Gemisch von Butter und vielleicht Schmalz, während die hohe Verseifungszahl in No. 15 und 16 sowie die anderen Konstanten auf einen hohen Cocosfettgehalt schließen Iassen. Bei No. 30, 31, 37 scheint eine Mischung von einem Teil Butter mit zwei Teilen eines anderen Fettes (Schweineschmalz?) vorzuliegen. No. 7,8 und 9 weisen einen sehr bohen Schokoladengehalt auf, sodaß das Fett hauptsächlich Kakaobutter ist, wozu auch die Konstanten ungefäbr stimmen. No. 10 dürfte ohne einen Fettzusatz gebacken sein und das Fett ausschließlich aus Eiern stammen. Der Lecithinphosphorsäuregehalt spricht für 5-6 Eier auf $500 \mathrm{~g}$ Mehl. Hierfür gibt Juckenack in seiner für Eierteigwaren berechneten Tabelle einen Ätherextrakt von 4,75 bis $5,45 \%$ an. Da bei diesem Gebäck, wie die Analyse zeigt, etwa die Hälfte des Teiges aus Mehl besteht, so ist der Fettgehalt von $4,63 \%$ in der Tat hauptsächlich auf das Eifett zurückzuführen. Die Konstanten sind gleichfalls die für das Eifett ermittelten. Bei No. 17 und 18 ist wohl allein Schweineschmalz verwendet worden.

1) Diese Zeitschrift 1900, 3, 1 .

2) Diese Zeitschrift 1914, 27, 761 . 
Im Anschluß hieran mögen auch einige Analysen von Backwaren mitgeteilt werden, die von dem Nahrungsmittel-Untersuchungsamt an der hiesigen Landwirtschaftlichen Versuchsstation ausgeführt und uns von dessen Vorsteher Herrn Dr. Kopp in dankenswerter Weise überlassen sind. Die Gebäcke wurden auf dem Wege der Nahrungsmittelkontrolle entnommen.

Tabelle VI.

\begin{tabular}{|c|c|c|c|c|c|c|c|c|c|c|}
\hline \multirow[b]{2}{*}{$\dot{8}$} & \multirow[b]{2}{*}{ Nähere Bezeichnung } & \multirow[b]{2}{*}{$\begin{array}{c}\text { Preis } \\
\text { für } \\
1 \mathrm{~kg} \\
\text { Mk. }\end{array}$} & \multirow[b]{2}{*}{ Wasser } & \multirow[b]{2}{*}{$\begin{array}{c}\text { Fett in } \\
\text { der } \\
\text { Trocken- } \\
\text { substanz } \\
\%\end{array}$} & \multicolumn{6}{|c|}{ Konstanten des Fettes } \\
\hline & & & & & 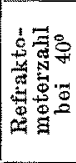 & 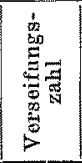 & 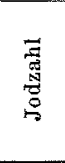 & 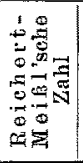 & 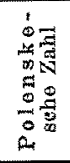 & 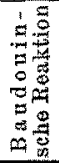 \\
\hline 1 & Buttergebäck J.E. in B. . & 1,00 & 13,13 & 31,05 & 54 & - & - & 0,61 & 0,60 & + \\
\hline 2 & Battergebäck F. D. in B. . & 2,50 & 11,15 & 24,41 & 45,5 & - & - & 21,90 & 1,65 & 0 \\
\hline 3 & Kaffeegebäck A.S. in B. & 2,00 & - & 31,08 & - & - & 一 & 23,54 & 3,20 & 0 \\
\hline 4 & Kaffeegebäck J. L. in M. . & 1,70 & 36,74 & 22,57 & 49 & - & 一 & 3,52 & 2,65 & + \\
\hline 5 & Butterwecken J. Kl. in M. & 0,80 & 28,41 & 5,21 & - & - & - & 3,27 & 2,40 & + \\
\hline 6 & Kachen W.S. in M. . . & 0,90 & 12,54 & 7,91 & 51 & - & 一 & 5,10 & 1,75 & + \\
\hline 7 & Butterwecken $\mathrm{J} . \mathrm{K}$. in $\mathrm{M}$. & 1,00 & 22,89 & 8,57 & - & - & - & 2,86 & 1,65 & + \\
\hline 8 & Englisch. Kuchen O.S. in M. & 2,20 & 10,83 & 16,35 & 46,5 & - & - & 23,98 & 3,80 & 0 \\
\hline 9 & Gebäck . . . . . . . & 1,00 & 23,08 & 6,25 & 55,8 & 195,9 & 60,0 & 1,66 & 0,70 & + \\
\hline 10 & Speknlatius (m, rein, Butter) & 1,20 & 7,72 & 10,58 & - & 201,5 & 69,4 & 2,95 & 2,50 & + \\
\hline 11 & Amerikan. Zwieback . & 1,40 & 18,77 & 8,09 & 58,1 & 199,0 & 64,8 & 4,19 & 2,10 & + \\
\hline 12 & Blätterteig-Gebäck . & 2,90 & 15,49 & 25,81 & 43 & 234,0 & 31,0 & 26,52 & 2,20 & 0 \\
\hline 13 & do. & 2,50 & 8,37 & 26,20 & 43,9 & 228,0 & 33,0 & 24,32 & 2,20 & 0 \\
\hline 14 & Weingebäck & 2,70 & 13,92 & 17,78 & - & 210,6 & 53,4 & 10,35 & 1,10 & 0 \\
\hline 15 & Blätterteig-Gebäck & 1,80 & 24,19 & 21,11 & 42,2 & $|233,0|$ & 28,0 & 27,62 & 2,60 & 0 \\
\hline
\end{tabular}

Von diesen Waren sind No. 2, 3, 8,12,13 und 15 mit Butter, ferner 14 wenigstens zum Teil mit Butter, die anderen mit Margarine gebacken. No. 1, 5 und 10 sind als "Butterwecken" oder unter der Bezeichnung "mit reiner Butter gebacken" "verkauft worden.

Die angeführten Untersuchungsergebnisse lassen also erkennen, daß man mit ziemlicher Sicherheit aus den Konstanten des geeignet gewonnenen Fettes die Natur des zur Herstellung des Gebäckes verwendeten Fettes feststellen kann, wenn man die Grenzen für die Konstanten etwas weiter nimmt als gewöhnlich; z. B. kann die Reichert-Meißl'sche Zahl 20,4 bei No. 22 noch ganz gut auf reine Butter hindeuten. Jedenfalls ist die Beantwortung der Frage, ob Butter oder ein anderes Fett vorliegt, stets möglich.

Es erhebt sich nun die Frage, inwieweit die Verwendung eines Ersatzfettes für die Butter zur Herstellung von Backwaren zulässig ist. Hierüber gehen die Ansichten der Sachverständigen und Gerichte vielfach auseinander. Ohne Frage ist es woh] stets als eine Täuschung des Publikums und als Nahrungsmittelfälschung zu betrachten, wenn ein Händler trotz eines Anschlages, „in seinem Betriebe werde nur Naturbutter verarbeitet", mit Butterersatzstoffen hergestellte Backwaren verkauft, mag es sich um „Buttergebäck“ oder anderes Gebäck handeln. Anders liegt die Sache, wenn ein Gebäck unter einem Namen verkauft wird, der das Wort Butter enthält, aber mit Butterersatzstoffen hergestellt ist, ohne dab dieses bekannt gegeben wurde. Die meisten Nahrungsmittelchemiker halten auch hier eine Nahrungs- 
mittelfälschung für vorliegend, wie aus fast allen Gutachten, die in der letzten Zeit zu derartigen Prozessen erstattet sind, hervorgeht. Auch die Handelskammer für das Großherzogtum Sachsen erkennt die Berechtigung dieses Standpunktes in einem Merkblatt für den Handel mit Nahrungs- und Genußmitteln an ${ }^{1}$ ): „Mit Margarine hergestellte Backwaren dürfen nicht als „Butterbackwaren" bezeichnet werden und letztere dürfen außer Butter und Butterschmalz keinen Zusatz anderer Fette erhalten".

Das Schöffengericht Erfurt erkannte in einem Falle auf Freisprechung, ,weil das kaufende Publikam nicht getăuscht worden sei, da es wisse, daß man für 10 Pfg. kein Buttergebäck verlangen könne, zu dem als Fettstoff ausschliefilich Butter verwendet sei, das Oberlandesgericht Kiel hat im Urteil vom 22. Dezember 1906 einen Freispruch der Strafkammer Altona in einem sehr schroffen Fall bestätigt: Ein Bäcker batte Kuchen, welchen Brothändler als "Butter- oder Blechkuchen" bei ihm käuflich bestellt hatten, mit Margarine hergestellt, unter der bestellten Bezeichnung geliefert, und zwar zum Preise von 1,60 Mk., während bei Verwendung von Butter das Gebäck 3 Mk. gekostet haben würde. Es sei erstens Branch in Altona, den sog. Butterkuchen nicht mit Butter, sondern mit Margarine herzustellen; dieser Brauch sei dem kaufenden Pablikum bekannt, es wisse, daß es beim Einkauf von Butterkuchen nur Kuchen aus Margarine erhalte. Wean dann durch die Verwendung von Margarine und der übrigen Zutaten unter Ausschlußs von Butter eine im gewöhnlichen Verkehr für normal gehaltene Beschaffenheit erzielt werde, könne der Kuchen nieht als verfälscht oder nachgemacht bezeichnet werden, da das Abweichen von der normalen Beschaffenheit wesentliches Merkmal eines verfälschten oder nachgemachten Nahrungš- und Genu官. mittels sei.

Ähnliche Urteile sind neuerdings durch mehrere andere Gerichte gefällt worden, so z. B. vom Schöfengericht Bunzlau vom 25. Nov. 1913 in einem Falle, wo ein Bäcker zu „Butterschnecken" statt Butter nur Margarine verwendet hatte. Die erste Strafkammer des Landgerichtes in Liegnitz hat dieses Urteil in der Sitzung vom 16. Januar 1914 bestätigt, weil der Bäcker das Gebäck nur als Sehnecken oder Zuckersehnecken feilgeboten und seine Fran den Verkauf besorgt habe.

Das Schöflengericht Neustadt a. d. Hdt. ist in der Sitzung vom 27. September 1913 ebenfalls zu einem Freispruch gekommen, weil vorausgesetzt werden könne, daß3 dem Publikum die Verwendung von Butterersatzstoffen in den Bäekereien bekannt sei und weil der Gutachter Prof. Dr. E. Schottelius geltend gemacht hatte, dak die Verwendung von Kunstspeisefetten (gemeint ist Margarine) anstatt von Butter durchaus gebilligt werden könne, da sie vielfach reiner und von besserem Geschmack seien als Butter. Die Strafkammer Frankenthal hat aber nach Zeitungsberichten das schöffengerichtliche Urteil in Neustadt a. d. H. aufgehoben und jeden der Angeklagten zu $20 \mathrm{Mk}$. Geldstrafe nebst Tragung der Kasten verurteilt. Eine gegen dieses Urteil eingelegte Revision wurde vom O.L.G. München am 31. März 1914 verworfen.

Der "Nahrungsmittel-Interessent" teilte in der Nummer vom 16. Februar 1914 noch folgenden Fall mit: Ein schlesisches Schöffengericht vertrat die Ansicht, daß3 Buttergebäck mit keinem anderen Fettstoff hergestellt werden dürfe als mit Butter. Die Strafkammer stimmte dem zu; das Oberlandesgericht Bresla u aber hob das Urteil auf, indem es ausführte, daß bei der Vorzüglichkeit maneher Margarinesorten tatsächlich gute Margarine ebenso wertvoll sei als schlechte Naturbutter.

Dieser auch vielfach von Gutachtern geltend gemachte Grund, um in der Verwendung von Margarine an Stelle von Butter, wo jedermann letztere erwartet, keine Verfälschung oder Nachmachung zu erblieken, erscheint aber nicht stichhaltig und muf zu unhaltbaren Zuständen führen.

1) Halbmonatsschrift für die volkswirtschaftlichen, gesetzgeberischen und kommerziellen Interessen der Margarineindustrie Düsseldorf 1911, 4, 275. 
Gezuckerter und nachgemachter Wein kann für den Nichtweinkenner ebenso gut schmecken und riechen, anch reiner, $\mathrm{d}, \mathrm{b}$. klarer sein und weniger Mikroorganismen enthalten als ein saurer und unklarer Naturwein, aber es wäre doch, ganz abgesehen davon, das das Weingesetz entgegensteht, unhaltbar, es als erlaubt ansehen zu wollen, daf3 ein nachgemachter, gefälschter oder Kunstwein, mit einer bestimmten Marke versehen, als Naturwein in den Verkehr gebracht werde. Nicht der Geruch und Geschmack sowie das äußere Ansehen allein bedingen den Wert eines Stoffes als menschliches Nahrungs- und Genufimittel, sondern anch seine sonstige Wirkung auf den Körper, seine Wirkung auf die Nerven, die Verdaulichkeit asw., Kigenschaften, die von seiner Wesensbeschaffenheit abhängig sind. Die Wesensbeschaffenheit von Butter und anderen Fetten ist aber sehr verschieden; die Butter enthält bei regelrechter Beschaffenheit neben spezifischen Glyceriden der niederen Fettsäuren besondere Aromastoffe, die allen anderen Fetten abgehen und die es ermöglichen, daß3 wir von der Butter Mengen verzehren und vertragen können, die uns von anderen Fetten widerstehen und nicht bekommen würden.

Diese Auffassung haben sich auch verschiedene Gerichte zu eigen gemacht, Das Landgericht $\mathrm{Ha} \mathrm{mburg}$ hat in der Sitzung vom 24. April 1904 die „Verwendung von Margarine zn Butterwaffeln" als eine Verfälschung im Sinne des $\$ 10$ Ziffer 2 des N.M.G. erklärt, "weil zwischen Butter und Margarine ein erheblicher Nährwert- und Preisunterschied bestehe und daf, wer eine ausdrücklich mit Butter hergestellte Ware verlange, eine mit Margarine bereitete nicht wolle."

Auch das Landgericht I Berlin hat in einem Urteil vom 1. August 1904 die Margarine für weniger wertvoll gehalten und einen „Schnecken- oder Napfkuchen“, zu dessen Herstellung $1 / 2$ Butter und ${ }_{1 / 2}$ Margarine verwendet war, als verfälscht erklärt, weil der Angeklagto am Schaufenster seines Ladens Plakate angebracht hatte, nach welchen seine Kuchen mit garantiert reiner Naturbatter hergestellt seien.

Einen gleichen Standpankt hat das Bayerische Oberste Landesgericht in verschiedenen Urteilen eingenommen (vergl. auch oben S. 782). In einem Urteil vom 15. Januar 1907 hat es in Übereinstimmung mit dem Berufungsgericht „Kaffeehörnchen", die mit Margarine zubereitet waren, im Sinne des $\$ 10,1$ und 2 d. N.M.G. für verfälscht erklärt, weil die Margarine im Vergleich mit Butter bzw. Butterschmalz minderwertig sei und ihre Verwendung auch dann nicht gerechtfertigt werden könne, wenn die Verwendung von Margarine bei Herstellung des mürben Brotes naheza als ein allgemeiner Geschäftsgebrauch anzusehen sei. Denn, so heißt es in dem Urteil, „Geschäftsgebräuche können, wenn sie den Zwecken des Gesetzes zuwiderlaufen, auch wenn sie in größerem Umfange geübt werden, dadurch niemals eine Berechtigung auf Bestehen oder Fortbestand erlangen".

In den Urteilen von 13. Juni 1908 und 23. Juni 1908 wurden beidemal die Revisionen verworfen, welche gegen die Verurteilungen des Berufungsgerichtes nach $\$ 10$ des N.M.G. eingelegt worden waren, nämlich in einem Falle, weil mürbes Gebäck, im anderen Falle weil Kaffeehörnchen mit Margarine und Palmin zubereitet worden waren. Das Bayerisch 0 Oberste Landgericht bestätigte in beiden Fällen ein Vergehen gegen $\$ 10$ des genannten Gesetzes.

Das Landgericht B a mberg hat in einem Urteil von 11. Januar 1906 die Verwendung von Rindstalg an Stelle von Butterschmalz zur Herstellung von mürben Brotwaren als Vergehen gegen $\S 10,1$ und 2 des N.M G. angesehen, weil Rindstalg gegenüber Butterschmalz minderwertig sei und das Pablikum die Ware nieht gekauft haben würde, wenn es von dieser Verwendung Kenntnis gehabt hätte.

Vom dem Schoffengericht in Heidelberg wurde in der Sitzung vom 3. Mai 1913 ein Bäcker, der bei der Herstellung von Buttergebäck $3 / 4$ Butter and $1 / 4$ Palmin verwendet hatte, wegen "Nachahmens" im Sinne des $\S 10$ N.M.G. und von der Strafkammer wegen Verfälschung verarteilt. Ein gleiches Urteil wird vom Sehöfengericht Dresden in einem Falle, in welchem es sich um Verwendung von Margarine za Butterzwiebäcken handelte, berichtet.

Angesichts dieser verschiedenen Anschauungen und Gerichtsentscheidungen erscheint es durchaus geboten, daß ebenso wie für die isolierten Fette als solche so auch 
für die Back- und Zuckerwaren gesetzliche bezw. amtliche Vorschriften, betreffend die hierzu anzuwendenden Fette, erlassen werden. NaturgemäB muß für alle Back- und Zuckerwaren, bei denen man von alters her oder nach der Bezeichnung das verwendete Fett als der Milch oder dem Rahm oder der Butter entstammend vorausseizen kann, die Verwendung eines anderen Fettes ohne Deklaration als Verfälschung gelten.

Dementsprechend hat der "Deutsche Milchwirtschaftliche Verein" unter dem 8. November 1912 an den Bundesrat eine Eingabe gerichtet, in der gefordert wird, daß Gebäcke, bei denen aus den zusätzlichen Worten "Butter", "Rahm" oder "Sahne" auf ihre Verwendung geschlossen werden könne, diese Stoffe auch wirklich enthalten müssen, und diesem Antrage haben sich viele Landwirtschaftskammern angeschlossen.

\title{
Nachweis von Albuminen, Gelatine und Agar-Agar in Sahne und Speiseeis.
}

Von

\begin{abstract}
J. König und W. Burberg.
Mitteilung a us der Landwirtschaftlichen Versuchsstation Münster i. W.
\end{abstract}

[Eingegangen am 7. Januar 1914.]

Sahne, Schlagsahne und Speiseeis erhalten mitunter Zusätze von Zuckerkalk, Weißei ${ }^{1}$, Gelatine, Agar-Agar, Gummi, Mehl und anderen Stoffen als Versteifungsoder Gelatiniermitteln. Der Nachweis von Mehl bietet keine Schwierigkeiten. Der von Zuckerkalk ist durch die Verfahren von E. Baier und $\mathrm{P}$. Neumann ${ }^{2}$ ), sowie von S. Rothenfußer ${ }^{3}$ ) ermöglicht. Über den Nachweis von Weißei, Gelatine, AgarAgar, Gummi im Speiseeis, die vielfach auch angetroffen werden, liegen aber bis jetzt noch keine Untersuchungen vor. Deshalb haben wir versucht, diese Lücke auszufüllen.

Die zur Herstellung des Speiseeises nötige Eismaschine besteht aus einem Kessel pon Zinn oder verzinntem Kupfer, der in einem starken Holzkübel drehbar ist. Die Drehung erfolgt gleichmäßig, entweder mit der Hand oder auf mechanischem Wege. Hierdurch soll erreicht werden, daß die Masse nicht zu einem harten Klumpen erstarrt und daß ein gleichmäßiges starres Erzeugnis erzielt wird. Der Raum zwischen Kessel und Holzkübel dient zur Aufnahme der Kältemischung (zerkleinertes Roheis + Viehsalz).

Nach den verwendeten Rohstoffen sind folgende Gruppen zu unterscheiden:

1. Cremeeise. Thr Hauptvertreter ist das Vanilleeis. Mehrere ganze Eier und Eigelbe werden mit Zucker schaumig gerührt, dazu kommt Milch oder Sahne und eine aufgeschnittene Vanilleschote. Die Masse wird unter ständigem Umrühren, um Anbrennen zu verhindern, auf dem Feuer nicht ganz zum Kochen erhitzt, durch ein Haarsieb in die Gefrierbüchse gegossen und gefrieren gelassen. In dieser Creme können natürlich alle möglichen anderen Zutaten vorhanden sein, sodaß Eise von ganz verschiedenem Geschmack entstehen; besonders. bekannt ist das Schokoladeneis.

2. Fruehteise. Sie werden durch Gefrierenlassen einer Masse aus frischem oder eingemachtem Fruchtmark, in Wasser gelöstem Zucker, unter Zusatz von Citronensäure her-

1) Hiermit sei das Weiße des Hühnereies bezeichnet.

2) Diese Zeitschrift 1904, 8, 605.

3) Diese Zeitsehrift $1909,18,135$ u. $1910,19,465$. 